\title{
Flap transplantation for treatment of pelvic fracture and defective hip
}

\author{
Bo Feng , Kecheng Niu, Zhi Zhang, Lan Zhang, Yunyan Tan \\ The Third Affiliated Hospital of Inner Mongolia Medical University, Hohhot, China
}

Received: June 13, 2012

DOI: $10.14725 /$ dcc.v1n1p33

\author{
Accepted: July 20, 2012 \\ Online Published: October 15, 2014 \\ URL: http://dx.doi.org/10.14725/dcc.v1n1p33
}

\begin{abstract}
A case of flap transplantation for treatment of pelvic fracture and defective hip in the Third Affiliated Hospital of Inner Mongolia Medical University was collected and analyzed on the basis of diagnosis, physical examination and treatment. The patient was in serious condition with large necrotic area and prone to lead to various complications. The paper aims to share experience and provide references for similar cases amongst surgeons.
\end{abstract}

Key Words: Pelvic fracture, Soft tissue defect, Skin flap transplantation

\section{Medical record}

A 24-year-old male patient was admitted to our hospital due to pain in waist, abdomen and hip accompanied by active bleeding in left hip and the right waist back (amount was unknown), activity limitation for 7 hours from a motor vehicle crash on March 12, 2012. He received transfusion of blood type A packed red blood cells with volume of $400 \mathrm{ml}$ without effective outcome in other hospitals prior to his admission. He was without any diet, urinary and bowel elimination since the occurrence of the disease. Abdominal ultrasound showed the right lower of the abdominal fluid sonolucent area. CT examination demonstrated the right transverse process fractures in 1-5 lumbar vertebrae, spinous process fracture in 4,5 lumbar, factures in left superior ramus of pubis and left ramus of ischium, and bilateral iliac bone fractures.

\section{Physical examination}

Data on the physical examination includes $\mathrm{T} 36.8^{\circ} \mathrm{C}, \mathrm{P}$ 95/min, R 23/min, BP 75/40 mmHg. He was conscious and showed poor in spirit, but fluent in the language. The heart rate was 95 beats per minute, showing regularity in the force and rhythm of the heartbeat. Double lung breath sounds resonance. Both abdominal tenderness and rebound tenderness and muscle tension were presented. No mass was seen in the abdomen. No enlargement of liver, spleen and kidneys beneath the rib was found. Murphy's sign was negative, shifting dullness was positive, and bowl sound was weaker. Skin contusion $(20 \mathrm{~cm} \times 20 \mathrm{~cm}$ in size $)$ in left hip and the right waist back was found. An open wound $(15 \mathrm{~cm}$ in length) in left hip and the right waist back with degloving injury, permeability and active bleeding in internal part was presented. Pelvic compression and separation test was positive. Palpable sense of bone rubbing and audible sense of bone crepitus were displayed. The double lambs showed adduction, outboard-rotating and flexion deformity, sensory in peripheral was normal and blood circulation was also normal.

\section{Laboratory examination}

Blood test: WBC $9.95 \times 10^{9} / \mathrm{L}, \mathrm{RBC} 1.76 \times 10^{12} / \mathrm{L}$, PLT $90 \times 10^{9} / \mathrm{L}, \mathrm{Hb} 53.0 \mathrm{~g} / \mathrm{L}, \mathrm{HCT} 15.7$. Stool routine: fe-

\footnotetext{
*Correspondence: Bo Feng, E-mail: nmgbgyyyy@ 163.com; Address: Department of Orthopedic, The Third Affiliated Hospital of Inner Mongolia Medical University, Hohhot, China
} 
cal's blood was positive; $\mathrm{PH} 7.2, \mathrm{PCO}_{2} 45.4 \mathrm{mmHg}, \mathrm{PO}_{2}$ $95 \mathrm{mmHg}$, BE $2.5 \mathrm{mmol} / \mathrm{L}, \mathrm{HCO}_{3}^{-} 17.6 \mathrm{mmol} / \mathrm{L}$. Four elements for transmitted: HBsAg negative, HCV-IgG negative, HIV negative, Anti-TP negative; $\mathrm{K}^{+} 5.13 \mathrm{mmol} / \mathrm{L}$, $\mathrm{Na}^{+} 141 \mathrm{mmol} / \mathrm{L}, \mathrm{Cl}^{-} 15 \mathrm{mmol} / \mathrm{L}, \mathrm{Ca}^{2+} 1.76 \mathrm{mmol} / \mathrm{L}, \mathrm{Glu}$ $12.6 \mathrm{mmol} / \mathrm{L}$; liver function: ALT $69 \mathrm{U} / \mathrm{L}$, AST $178 \mathrm{U} / \mathrm{L}$, CK 9,650 U/L, LDH 654 U/L, HBDH 364.0 U/L, blood coagulation: TP $40.1 \mathrm{~g} / \mathrm{L}$, PT $29.8 \%$, APTT 45.9 s, TT $21.6 \mathrm{~s}$.

\section{Primary diagnoses}

(1) Hemorrhagic shock

(2) Diffuse peritonitis

(3) Blunt abdominal trauma: Visceral ruptures?

(4) Fracture of transverse process in the right side of 1-5 centrum of lumbar vertebra

(5) Fracture in lumbar 4,5 spinous process

(6) Bilateral iliac bone comminuted fracture

(7) Fracture in the left superior ramus of pubis and ischial ramus

(8) Degloving injury in the left hip and right waist back, contusion damage in skin and soft tissue, muscle rupture(see Figure 1, Figure 2)

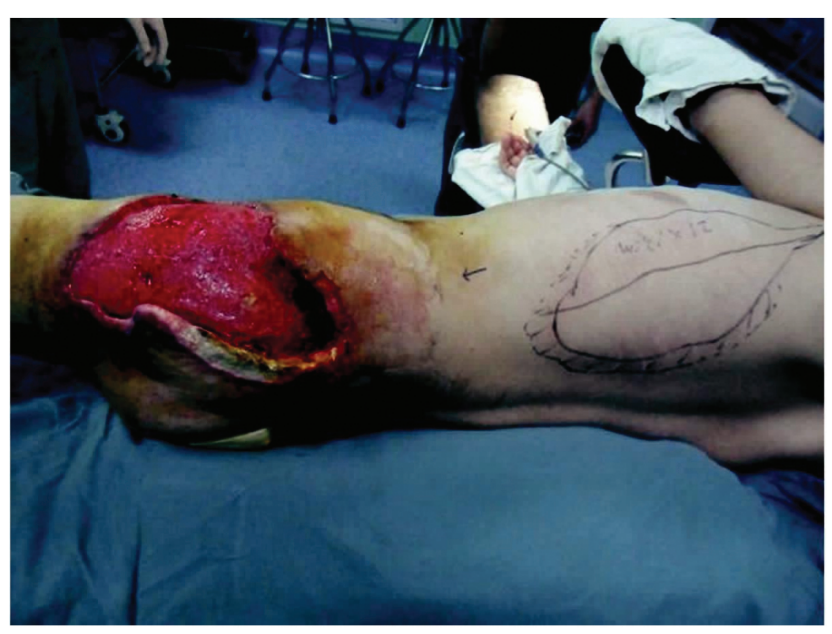

Figure 1: Preoperative skin necrosis and defect area

\section{Treatment and diagnosis}

The patient received packed red blood cells transfusion against shock and maintained the balance of fluid, electrolytes and acid-base since he was admitted to our hospital. An exploration of his abdomen found about $500 \mathrm{ml}$ fresh bloody liquid within abdominal cavity, and confirmed the normal of stomach, pancreas, liver, gallbladder and spleen. Rupture of small blood vessels was visualized in mesocolon and they were ligated in due course. Luckily, no ruptures of the bowl could be seen in the examination. Besides that, retroperitoneal hematoma was timely treated with rubber drainage tube in the pelvic floor.

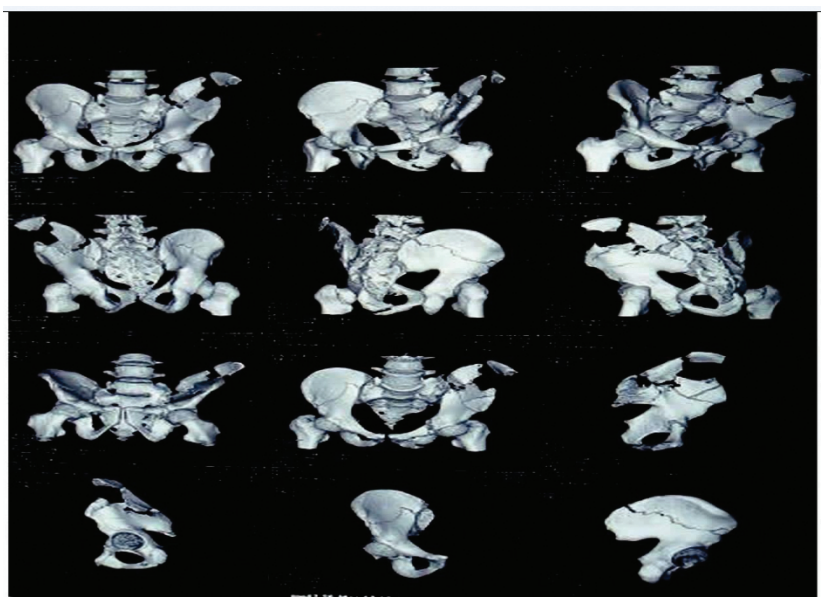

Figure 2: Preoperative three-dimensional reconstruction

Examination results included: an open wound $15 \mathrm{~cm}$ in length on the outside of left anterior superior iliac spine, active bleeding, degloving injury in skin $(20 \mathrm{~cm} \times 20 \mathrm{~cm}$ in size), contusion and fracture of gluteus maximus, gluteus medius, gluteus minimus and piriformis, gluteal artery and vein were fractured in the upper parts of piriformis and were respectively be ligated. Comminuted fractured of left iliac bone and sacrum were untreated till the second surgery. During the surgery, the patient underwent reposition for sacral fractures, electric coagulation hemostasis for fractured intermuscular vessels and suture for fractured muscle. Three negative pressure drainage tubes were placed into the pelvic floor, sutured layer by layer, intraoperative bleeding were about $1,200 \mathrm{ml}$, so a corresponding volume of packed red blood cell was infused. The patient was transferred to intensive care unit and general surgery department after the surgery. He was in stable condition with the symptomatic treatment of anti-inflammatory and the drainage tube was pulled out then. The patient turned to our department for further treatment on March 24, 2012. Examination result in our department showed necrosis of buttock skin and muscle $(20 \mathrm{~cm} \times 15 \mathrm{~cm}$ in size $)$.

Consequently, a change of medical prescription together with anti-inflammatory was performed given that situation. The patient had a second surgery under general anesthesia on March 29, 2012. Pelvic structure, degloving injury in the small of the back and soft tissue contusion are the main symptoms that should be dealt with during the surgery. Then, the patient underwent a series of operation, such as necrosis debridement, debridement, vacuum sealing drainage (VSD), open reduction and internal fixation by plates and screws. He was taking cefuroxime and etimicin sulfate for anti-infection after the second surgery. In addition to that, VSD was changed every seven days, as a result, the patient recovered well without infection and 
necrotic granulation grow very well. He was scheduled for flap coverage on the basis of general discussion. The flaps which were available for the surgery were (1) free latissimus dorsi musculo-cutaneous flap, (2) predicled anterolateral skin flap, (3) rectus femoris flap and (4) tensor fascia lata myocutaneous flap. The selection of flap depends on the real circumstances. On April 27, 2012, muscle necrosis in left hip was found and soft tissue defect of skin was presented. Therefore, reversed rectus femoris flap repair surgery was performed. Firstly, took the skin flap from the left thigh, leaving the residual wound area to be $20 \mathrm{~cm} \times 15$ $\mathrm{cm}$ in size and ilium was exposed. Then, removed the necrosis organizations around the wounds, closed part wound, opened each layer along the front of thigh to display rectus femoris. Protected arteria circumflexa femoris late-ralis and rectus femoris when seperating rectus femoris. The skin flap was about $12 \mathrm{~cm} \times 8 \mathrm{~cm}$ in size with good blood circulation. Took proximal end as axis rotation to suture the damaged parts of left hip skin and soft tissue layer by layer. Got the skin grafts from the thigh in case some parts could not be stitched. The respectivesuture was performed in the two wounds of anterior superior iliac crest and skin necrosis of waist and back after debridement. The patient took Dextran 40 and amino acids injection, papaverine, low molecular weight heparin for vasodilation, received anticoagulant therapy, continuously diathermy for insulation, irrigation drainage catheter, and anti-inflammatory treatment At present, he was in stable condition with good blood circulation of skin flap. He was taking rehabilitation training and body functions were gradually recovered.

\section{Discussion}

\subsection{Dr. Kecheng Niu}

Dr. Kecheng Niu is a doctor in the Third Affiliated Hospital of Inner Mongolia Medical University, specializing in hand and foot surgery.

There have been many reports ${ }^{[3,4]}$ about the study of the tensor fasciae latae ever after Hill applied tensor fasciae latae free musculocutaneous flap transplant for the treatment of leg and foot ulcers and achieved success. ${ }^{[1,2]}$ The advantage and disadvantage of the treatment are as follows: It is an effective and safe treatment for soft tissue defect and bone exposure due to its abundant blood supply, strong antiinfection and fast recovery. Because of special requirement the factors of cover appearance in wound repair, repaired skin pressure resistance as well as skin feeling should be taken into consideration. Since the donor parts of this skin flap was hidden, the resection of the skin flap has less impact on limb function and appearance. Besides that, moderate thickness and large resection part are also the significant strengths of the treatment. Most importantly, it had sensory nerve, less incidence of vascular anatomical variation, longer predicle and thicker diameter, which guarantees the best outcome of the surgery. Moreover, it has greatest outcome at lower cost for its less pain, quick recovery, clear anatomical level, etc. Due to the limited source of muscle in the distal end of tensor fasciae latae, it could not fill the necrosis of the cavity. The selection of the treatment during the surgery depends on the real situation.

\subsection{Dr. Lan Zhang}

Dr. Lan Zhang is a doctor in the third affiliated hospital of Inner Mongolia Medical University, specializing in the diagnosis and treatment of common diseases in hand surgery.

In 1984, Da-chuan $\mathrm{Xu}^{[5]}$ and Li-sheng Luo ${ }^{[6]}$ firstly reported that the anatomy and clinical application of predicled anterolateral thigh flap with descending branch of lateral circumflex femoral artery. It has found its wide clinical application for soft tissue defect reconstruction for its concealed position, no cost of trunk blood vessel and simple operation, and has achieved better outcome. However, it must be resected together with fascia lata, which results in prolonged surgical time, suture difficulty, ischemic necrosis of the suture site, extensive treatment time and addition of hospitalization expenses. Therefore, a further exploration of its clinical application is warranted. Koshima ${ }^{[7]}$ found a wider perforator branch within anterolateral thigh. It has a wide support range, and the maximum range of skin flap was up to $35 \mathrm{~cm} \times 25 \mathrm{~cm}$. So perforator flap was widely in use since then. Its advantage lies in the fact that the resection of perforator flap only takes along fascia lata within $3 \mathrm{~cm}$ in range without causing damage of perforator flap vessel and maintains good blood supply. The advantages of anterolateral thigh flap are as follows: (1) It has rich blood supply and strong ability of anti-infection and it makes the cavity filling flexible and perfection. (2) The muscle flap and the skin flap were arranged in parallel so that it could broaden coverage area of the wound. In other words, the defect was provided with double the area of tissue flaps, which contributes to a perfect coverage of the wound on the thigh. On one hand, muscle flap plays an important role in expanding coverage area of skin flap. On the other hand, skin flap guarantees the blood supply of muscle flap. (3) Muscle flap could be well spread together with its muscle atrophy after denervation contributes to good appearance in the later stage. Therefore, the shape of muscle flap has advantages over skin flap amongst fatter patients. However, muscle flap and tissue of skin graft area were all tough. (4) Wound repair and functional reconstruction could be completed at the same time when cutting dynamic muscle flap. (5) Blood supply to medial half of vastus lateralis was a single trunk, and it is segmental blood supply to outer half of vastus lateralis. So the muscle contains extensive vascular anastomosis. There was a rich blood supply for the resectional muscle flap and reserved muscles. The independence of good nerve 
bundle makes it possible to cut dynamic muscle flap without causing any damage to power function of retained muscle. The disadvantages of anterolateral thigh flap are as follows: (1) This treatment method was not applicable for small defects. It found its wide clinical application for large and complex defects since the surgery is difficult. Small defects could be treated with other methods. (2) The anatomy of descending branch of the lateral artery of the knees could be very challenging in that anastomosis of distal vascular pedicle was required. (3) Solid microsurgical techniques are essential to perform anastomosis of arteries and veins. (4) The surgery owns some risk to a certain extent for doctors with less experience due to distribution of anterolateral vascular with potential variation.

\subsection{Dr. Zhi Zhang}

Dr. Zhi Zhang is the deputy Chief surgeon of the Orthopedic department at the third affiliated hospital of Inner Mongolia Medical University, specializing in trauma surgery and joint surgery.

In 1983, Dler reported six successful transfer of pedicled rectus femoris muscle flap for abdominal wall and hip defect, and the surgery provided morphological basis for the clinical application. The total length of the rectus femoris is different depending on the individual. The average length is $38.79 \mathrm{~cm} \pm 0.33 \mathrm{~cm}$. The beginning length of the muscle tendon is $5.55 \mathrm{~cm} \pm 0.16 \mathrm{~cm}$, the average width is $1.33 \mathrm{~cm}$ $\pm 0.04 \mathrm{~cm}$, the average thickness is $0.68 \mathrm{~cm} \pm 0.02 \mathrm{~cm}$ and the ending length of the musele tendon is $4.44 \mathrm{~cm} \pm$ $0.11 \mathrm{~cm}$, the average width is $1.41 \pm 0.04 \mathrm{~cm}$, the average thickness is $0.35 \mathrm{~cm} \pm 0.01 \mathrm{~cm}$. Muscle belly, the average length is $28.30 \mathrm{~cm} \pm 0.35 \mathrm{~cm}$, the average width is $3.29 \mathrm{~cm}$ $\pm 0.05 \mathrm{~cm}$, and the average thickness is $1.10 \mathrm{~cm} \pm 0.03 \mathrm{~cm}$. It functions to flex the thigh at the hip joint and to extend the leg at the knee joint. The rectus femoris, sartorius, and iliopsoas are the flexors of the thigh at the hip. It is a weaker hip flexor when the knee is extended since the action will recruit more iliacus, psoas major and tensor fasciae latae.

Similarly, the rectus femoris is not dominant in knee extension. So there is no obvious impact on the function of knee extension after the removal of the rectus femoris. Besides that, it is without much difficulty to resect rectus femoris due to its superficial location and loose binding with vastus medialis, vastus lateralis and the vastus intermedialis. Neurovascular bundle of rectus femoris is regular and entry point of femoral nerve and vessel branches to rectus is constant. There are two brunches of neurovascular bundle. The first group of vessels could be ligated as they are short and thin, which is not suitable for anastomosis. Therefore, anastomosis with thicker and longer vessels is required to ensure blood supply of rectus femoris. As a matter of fact, the two groups are the branches of nerve trunk. Anastomosis with nerve trunk and preserve all the nerves are, therefore, con- ducive to the recovery of muscle function. On one hand, rectus femoris is available for free transplant. On the other hand, vessels and nerves are in the proximal location. Next, proximal part of the neurovascular pedicles as the center, rotate the rectus femoris muscle flap to any direction. Moreover, rectus femoris carries the features of good blood supply, fast healing of wound and better outcome especially for hip defect which was caused by latent infection. The tips that the surgeons should bear in mind when they were cutting flap pedicled with descending branch of lateral femoral circumflex artery. Rectus femoris should be protected so as not to affect its blood supply and innervation given that it has one blood vessel and single nerve. In this case, reversed island flap was applied instead of free flap as it is close to rectus femoris muscles and part muscle could be used to fill necrosis cavity, which lowers the surgical risk and improves the ability of musculocutaneous flap to resist infection. The patient underwent internal fixation of pelvic fractures in the early stage, which provides a good basis for the next flap surgery (see Figure 3, Figure 4).

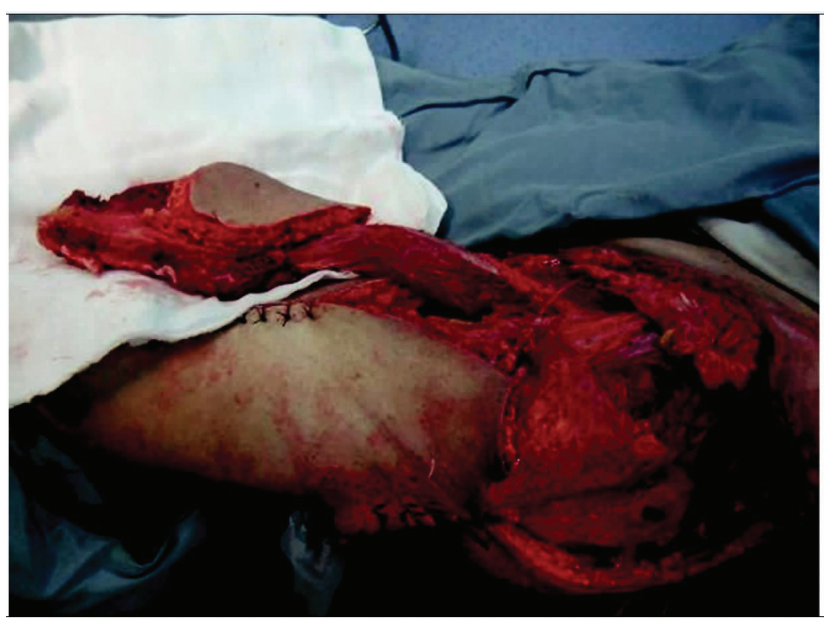

Figure 3: Separating rectus muscle flap

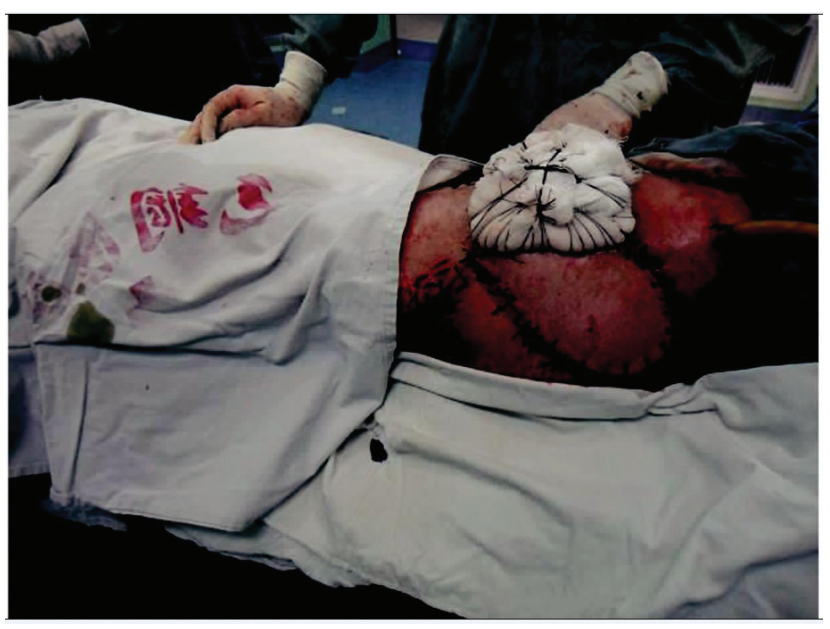

Figure 4: The rectus femoral flap covering the wound 


\subsection{Dr. Yun-yan Tan}

Dr. Yun-yan Tan is a head nurse of hands and feet surgery at the third affiliated hospital of Inner Mongolia Medical University, specializing in nursing care for skin flap surgery.

Effective nursing is crucial following successful skin flap surgery. Therefore, in addition to close monitoring of life indicators, observation of blood volume requires more attention since rich blood volume is beneficial to blood supply of skin flap. ${ }^{[11]}$

Postoperative observation of peripheral circulation of skin flap in the earlier stage carries more weight: (1) Close observation of the color on local skin flap. It means arterial blood supply is good when the skin flap is presented with rosy color. On the contrary, it is presented with pale color. Cyanotic color suggests inhibited venous return. (2) Close observation of capillary refill time. Normal capillary refill time is usually less than $3 \mathrm{~s}$. A prolonged capillary refill time (> $5 \mathrm{~s}$ ) may be a sign of circulation problem. Skin temperature is another objective indicator of capillary supply. The commonly average core skin temperature is over $31.0^{\circ} \mathrm{C}$. It reveals circulatory disturbance when the temperature is lower than $27.0^{\circ} \mathrm{C}$. (3) Close observation of local edema and hemorrhage. Flap anastomose site requires more attention to determine whether hemorrhage is from angiorrhexis. Confirm the cause for severe local edema whether it is poor reflux or inflammatory infection. Edema is generally improved within three days after the surgery. So keeping abreast of the local situation plays an important role in catching the sign of survival of flaps.

Nursing principles of the patients undergoing skin flap surgery: (1) Make sure the patient's psychological wellbeing so that he could get coordinate with the treatment. (2) Partial comfortable immobilized package. Plaster fixation for 3-4 weeks to keep skin flap from injuries of physical activities. (3) Make sure that supply of the skin flap transplantation is not affected. (4) Patient with vascular pedicle should avoid blood vessel distorting. (5) Drainage and partial drainage.

Nursing measures for implementation includes: (1) The patient was in a modified supine position and his injured limb was raising for $10 \mathrm{~cm}-20 \mathrm{~cm}$ above heart level to facilitate the swelling. (2) Avoid pressure from upper or lower extremities, such as upper limb extensor and lateral flexion of the lower limb. Plaster is needed to fix if necessary and limb is suspended. (3) Avoid vasospasm. Prevent the patient from painful stimuli, maintain comfortable limb immobilization, treat ischemia in due course and keep warm against cold. (4) Abstinence from bad alcohol, tobacco or spicy food that could stimulate vasospasm. (5) Use of antispasmodics drugs, such as tolazoline, aspirin and other blood vessel medicine. (6) Treatment for suspected poor thrombosis blood circulation is to keep warm against spasmolysis. Strive hard to establish revascularization within 6 hours.
(7) Anti-infection. Strict disinfection of indoor air and application of antibiotics at the early phrase.

Nursing measures at the end of the treatment. The patient endured severe damage from pelvic fracture. Hence, it is of great importance to carry on rehabilitation exercise for limb function after the confirmation of survival of skin flaps was made. Encourage patients to engage outdoor activities which can facilitate the expansion of blood vessels and exercise of lower extremity muscle.

\subsection{Dr. Bo Feng}

Dr. Bo Feng is an orthopedic physician at the third affiliated hospital of Inner Mongolia Medical University, specializing in hands, feet and ankles surgery and trauma surgery.

The patient's condition was complex as comminuted fractures of the pelvis occurred in conjunction with soft tissue defect of hip skin, exposure bone and severe wound infection. His condition was beginning to stabilize without signs of wound infection under the examination for abdomen exploration, fractures with internal fixation, wound debridement, granulation tissue covered by VSD and anti-infection therapy, which laid a solid foundation for flap repair surgery. The following treatment was to repair soft tissue defects and control infection. However, the significant issue, at present, for us was to choose appropriate repairing method. Factors involved in selecting musculocutaneous flap for the surgery include effectiveness, lower trauma and high success rate. Given the situation that contralateral back and waist suffered the same injury, flaps that were applicable for the surgery included free latissimus dorsi musculocutaneous flap, pedicled rectus femoris muscle flap, the tensor fascia latae musculocutaneous flaps and anterolateral thigh musculocutaneous flap. The latissimus dorsi musculocutaneous flap is one of the most versatile and reliable flaps available for use in reconstructive surgery and has gained widespread popularity in clinical application. It was firstly described by Tansini in 1896. In 1912, D'Este ${ }^{[12]}$ applied latissimus dorsi island flap to repair chest wall defect in a case of breast cancer resection operation, which turned out to be very successful. There had been many reports of similar treatment methods of latissimus dorsi island flap to cover chest abdominal wall defects and the hip defects since then. ${ }^{[13]}$ Schotstaedt $^{[14]}$ applied latissimus dorsi island flap to repair shoulder and the upper arm soft tissue defects. Bostwick ${ }^{[15]}$ reported the use of the predicled latissimus dorsi myocutaneous reversed island flap with intercostal artery for reconstruction in the soft tissue defects of the spine. Latissimus dorsi musculocutaneous flap received more attention ever after and was regarded as an optimal treatment for clinical practice.

Gross anatomy of latissimus dorsi muscle is as follows. Latissimus dorsi is v-shaped muscle and its upper edge is 
$200 \mathrm{~mm} \pm 23 \mathrm{~mm}$, inside edges of length is $245 \mathrm{~mm} \pm$ $32 \mathrm{~mm}$, lateral border is $337 \mathrm{~mm} \pm 36 \mathrm{~mm}$. Blood supplies to the muscle are mainly from thoracodorsal vessels, secondary segmental vessels and intercostal vessels. ${ }^{[16]}$ The dominant blood supplies are the thoracodorsal artery, the descending branch of transverse cervical artery and intercostal arteries which are branches of the subscapular artery. It is $21.6 \mathrm{~mm} \pm 17.0 \mathrm{~mm}$ in length and thoracodorsal artery is $71.4 \mathrm{~mm} \pm 25 \mathrm{~mm}$ in length. All these arteries are accompanied by veins. The thoracodorsal nerve, $96.2 \mathrm{~mm}$ $\pm 19.2 \mathrm{~mm}$ in length, is from posterior cord of brachial plexus, accompanied by thoracodorsal artery, and appears in the back of the axillary sheath. Rowsel, et al. ${ }^{[17]}$ obtained the same conclusion as Angrigiani by corrosion specimens and found direct perforating branch existed in 81 percent of the 100 specimens, its largest blood supply range was up to $25 \mathrm{~cm} \times 15 \mathrm{~cm}$ in size.

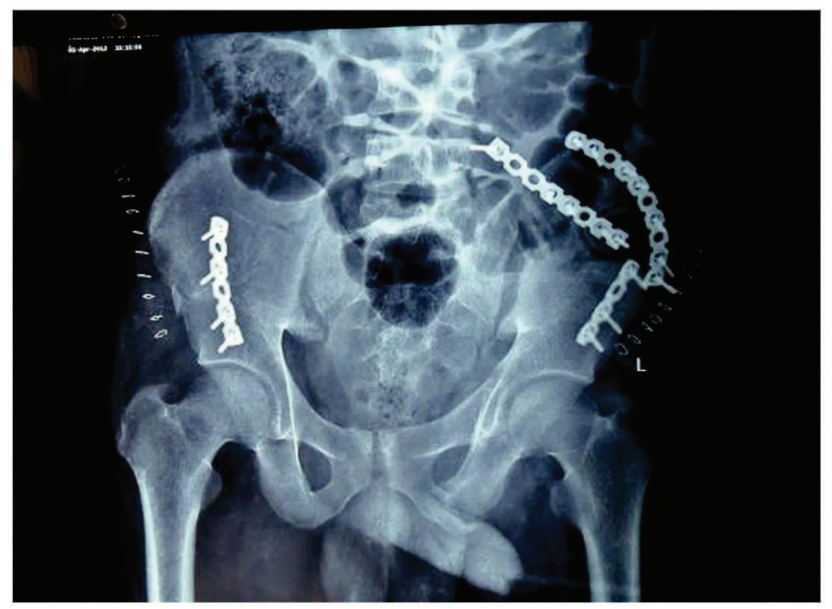

Figure 5: Internal fixtion of fracture

The advantage and disadvantage of latissimus dorsi musculocutaneous flap are as follows. The external diameter of the blood vessel is more than $1.5 \mathrm{~mm}-2.0 \mathrm{~mm}$, vascular pedicle of transplanted flap is up to $6 \mathrm{~cm}-8 \mathrm{~cm}$ and the skin that is available for transplant is up to $8 \times 20 \mathrm{~cm}-23 \times 40 \mathrm{~cm} .^{[18]}$ Furthermore, the donor area is hidden, the skin flap is of good quality, surgery operation is simple, and blood supply is very rich without any cost of vital blood supply artery. It is especially applicable for exposed bone infection and chronic osteomyelitis wound reconstruction as it has thick muscle flap with good reconstruction appearance, owns rich blood supply, and carries strong ability of anti-infection. The muscle flap is particularly for wound repair of merged dynamic muscle defect as it could initiate the dynamic muscle function at the first reconstruction. Though dysfunction in the donor area was not obvious, it also has a synergistic role in extension and lateral flexion of the lumbar spine, and assists as a muscle of both forced expiration and accessory muscles of ispiration. Therefore, the latissimus dorsi flap transplantation may cause great damage to donor area. Functions of latissimus dorsi are completely lost and shape of skin flap is bloated. Hence, follow-up repair is required when it is for face and neck, limbs or joint shallow wound reconstruction. In this case, the patient was presented with buttock skin soft tissue defects. There was no need for follow-up repair surgery. Thus, he fitted under the surgery. However, larger cutting area for the latissimus dorsi muscle may further aggravate injury.

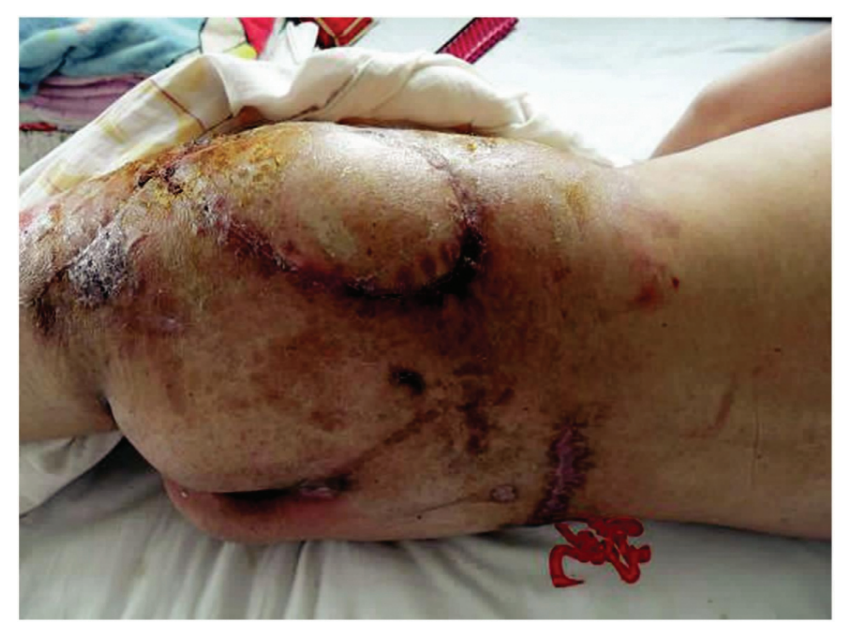

Figure 6: Six weeks after flap surgery

The descending branch of lateral femoral circumflex artery was found well and reversed island flap of rectus femoris was employed in the surgery. Muscle flap area of $15 \mathrm{~cm}$ $\times 10 \mathrm{~cm}$ in size, skin flap area of $10 \mathrm{~cm} \times 10 \mathrm{~cm}$ in size and muscle flap pedicle of $15 \mathrm{~cm}$ in length were transplanted during the surgery. The surgery is very successful with a complete survival of skin flap and controlled infection. The patient got better recovery under proper nursing measure. It provided experience for the future patients (see Figure 5, Figure 6).

\section{References}

[1] Hill HL, Nahai F, Vasconez LO. The tensor fascia latamyocutaneous free flap. Plast Rescontr Surg. 1978 Apr; 61(4): 517-22. PMid: 345303. http://dx.doi.org/10.1097/ 00006534-197804000-00004

[2] Yuefeng Qu, Moying Zheng. Application of tensor fascia lata flap graft repair of skin and soft tissue defect in ankle. Chinese Journal plastic surgery. 2009; 19(6): 409.
[3] Kunxiang Liu, Qian Xue, Mingyang Li. The fascia lata study on the neural point position. Acta Academiae Medicinae Zunyi. 2001; 24(5): 386-387.

[4] Yuebin Qiao, Chengjun Song, Songhe Yang, et al. The fascia lata applied anatomy of free muscle flap. Journal of Chengde Medical College. 2006; 23(01): 87-88.

[5] Dachuan Xu, Shidao Zhong, Muzhi Liu, et al. Anatomy of anterolateral femoral flap. Journal of Clinical applied anatomy. 1984; 2(3): 158-160. 
[6] Lisheng Luo, Jianlhua Gao, Linfeng Chen, et al. Anterolateral femoral skin flap and its application of graft. Journal of First Military Medical University. 1984; 4(1): 1-4.

[7] Koshima I, Yamamoto H, Hosoda M, et al. Free combined compositeflaps using the lateral circumflex femoral system for repair of massivedefects of the head and neck regions: An introduction to the chimeric flapprinciple. Plast Reconstr Surg. 1993; 92(3): 411-420. PMid:8341739. http://dx.doi.org/10.1097/ 00006534-199309000-00004

[8] Huiren Liu, Dequn Liu, Xiaopo Liu, et al. Leaves of anterolateral femoral flap applied anatomy and clinical significance. Chinese Journal of clinical anatomy. 2008; 26(5): 491-492.

[9] Shenghua Chen, Dachuan Xu, Xiaobin Zhou, et al. Descending branch of lateral femoral circumflex artery pedicled anterolateral thigh muscles of anatomic study on the design of flap. Chinese Journal of Clinical Anatomy. 2010; 28(3): 237-238.

[10] Ger R, et al. Tlie prevention and repair of large abdomenal - wall defeers by museletrans - Position: A Prelirninary eommunieation. Plaot eeonstrSurg. 1953; 72: 170.

[11] Haiming Li, Qingxia Jia, et al. Nursing care of limbs after flap surgery. Journal of Practical Orthopedics. 2005; 11(5): 478-479.
[12] D'Este S. La technique de l' amputation de la mammelle pourcarcinoma mammaire. Rev Chir. 1912; 45: 164-165.

[13] Luping Wang, Jiasheng Dong. Anatomical Study of the latissimus dorsi muscle flap and its clinical application progress. Journal of tissue engineering and Reconstructive Surgery. 2008; 4(2): 119-120.

[14] Schottstaedt ER. Complete muscle transposition. J Bone JointSurg. 1955 Oct; 37-A(5): 897-918, discussion, 918-919.

[15] Bostwick J. Sixty latissmus dorsi flaps. Plast Reconstr Surg. 1979 Jan; 63(1): 31-41.

[16] Mathes SJ, Nahai F. Classification of the vascular anatomy of muscles: Experimental and clinical correlation. Plast ReconstrSurg. 1981 Feb; 67(2): 177-187. PMid:7465666. http://dx.doi.org/ 10.1097/00006534-198167020-00007

[17] Rowsell AR, Eisenberg N, Davies DM, et al. The anatomy of thethoracodorsal artery within the latissimus dorsi muscle. Br J Plast Surg. 1986 Apr; 39(2): 206-209. http://dx.doi.org/10.1016/ 0007-1226 (86) 90083-4

[18] Chunlin Hou, Yudong Gu. FLAP surgery. ShangHai: Shanghai Science and Technology Press; 2006: 9p. 